\title{
Thoracic Endovascular Aortic Repair for Cardiopulmonary Arrest Due to Aortic Dissection
}

\author{
Yoshiro Kikuoka1, Naoki Fujimura ${ }^{2}$, Yu Michiura', Tomohiro Kamagata', Yumi Tsuchiya ${ }^{1}$, \\ Shiho Irino1, Motojiro Takebe ${ }^{1}$, Yoko Sugawara ${ }^{1}$, Satoshi Ohtsubo², Kazuhiko Sekine ${ }^{1}$
}

${ }^{1}$ Department of Emergency and Critical Care Medicine, Tokyo Saiseikai Central Hospital, Tokyo, Japan

${ }^{2}$ Department of Cardiovascular Surgery, Tokyo Saiseikai Central Hospital, Tokyo, Japan

Email: kikuokayoshirou@gmail.com

How to cite this paper: Kikuoka, Y., Fujimura, N., Michiura, Y., Kamagata, T., Tsuchiya, Y., Irino, S., Takebe, M., Sugawara, Y., Ohtsubo, S. and Sekine, K. (2021) Thoracic Endovascular Aortic Repair for Cardiopulmonary Arrest Due to Aortic Dissection. Case Reports in Clinical Medicine, 10, 387-392.

https://doi.org/10.4236/crcm.2021.1011048

Received: October 12, 2021

Accepted: November 14, 2021

Published: November 17, 2021

Copyright $\odot 2021$ by author(s) and Scientific Research Publishing Inc. This work is licensed under the Creative Commons Attribution International License (CC BY 4.0).

http://creativecommons.org/licenses/by/4.0/

(c) (i) Open Access

\begin{abstract}
Background and Aim: Reports on recovery from Stanford type A aortic dissection (TAAD) leading to cardiopulmonary arrest (CPA) are few. In retrograde TAAD (r-TAAD) cases, some authors reported the efficacy of thoracic endovascular aortic repair (TEVAR). However, only a few reports chose TEVAR for the treatment of $r$-TAAD resulting in cardiac arrest before hospital arrival. We report a case of $\mathrm{r}$-TAAD presenting with cardiac arrest before hospital arrival not indicated for surgery but TEVAR as treatment. Case: A 65-year-old woman with a history of Marfan syndrome presented to the emergency department after a CPA. Sequential return of spontaneous circulation was achieved $27 \mathrm{~min}$ after CPA. Contrast-enhanced computed tomography showed retrograde $\mathrm{r}$-TAAD with an entry tear to the false lumen in the thoracic descending aorta. Therefore, thoracic endovascular aortic repair (TEVAR) was performed with r-TAAD. Afterward, the clinical course was stabilized. This patient suggests that TEVAR is an effective option for the treatment of patients with hemodynamically unstable r-TAAD, even after CPA. Conclusion: TEVAR can lead to a successful recovery from cardiac arrest due to $r-T A A D$.
\end{abstract}

\section{Keywords}

Acute Aortic Dissection, Cardiac Tamponade, Cardiopulmonary Arrest on Arrival, Retrograde Stanford Type A Aortic Dissection, r-TAAD, Thoracic Endovascular Aortic Repair, TEVAR

\section{Introduction}

Reports on recovery from Stanford type A aortic dissection (TAAD) leading to 
cardiopulmonary arrest (CPA) are few, with a very poor prognosis [1]. Especially for the treatment of CPA due to TAAD, a fatal or unfavorable outcome is associated with CPA, coma, and stroke; therefore, surgery may not be performed from presuming poor prognosis [2]. However, some patients achieve good outcomes with surgery, even after the occurrence of CPA preoperatively [3]. Patients with CPA should not be routinely excluded from surgical indications [3].

Generally, surgery is the first treatment choice for TAAD with cardiac tamponade [4]. Retrograde TAAD (r-TAAD) is a TAAD subgroup with an entry tear at the descending thoracic aorta. In these cases, some authors reported the efficacy of thoracic endovascular aortic repair (TEVAR) [5]. However, only a few reports chose TEVAR for the treatment of r-TAAD resulting in cardiac arrest before hospital arrival.

Herein, we report a case of $\mathrm{r}$-TAAD presenting with cardiac arrest before hospital arrival not indicated for surgery but TEVAR as treatment.

\section{Case Report}

A 65-year-old woman with Marfan syndrome suffered from sudden back pain with numbness in both legs, which did not improve on rest. Therefore, an emergency medical service (EMS) was called ( $22 \mathrm{~min}$ before arrival hospital). The patient's condition worsened and eventually showed no response before the EMS arrival. Cardiopulmonary resuscitation (CPR) was not performed by a bystander until the emergency medical personnel arrived. When the ambulance arrived, her breathing ceased and pulseless electrical activity (PEA) was observed (11 min before hospital arrival). On hospital arrival, the patient was still in PEA state under EMS resuscitation. At 5-min after hospital arrival, the patient was successfully resuscitated with return of spontaneous circulation (ROSC) after the administration of $1 \mathrm{mg}$ of epinephrine, presuming a 27-min cardiac arrest (Figure 1) (5 min after hospital arrival).

Echocardiography revealed a large amount of pericardial effusion. Neither obvious aortic regurgitation nor abnormal wall motion was observed. Contrast-enhanced computed tomography (CT) scan revealed acute aortic dissection from the ascending aorta to the renal arteries with pericardial effusion (Figures $2(a)-(c))$ and an entry tear of the false lumen in the thoracic descending aorta (Figure 2(b) and Figure 2(c)). Head CT showed no signs of bleeding or severe ischemia.

Based on these findings, cardiac arrest was found to be caused by r-TAAD with cardiac tamponade with an entry tear located in the descending thoracic aorta. As hemodynamics remained unstable due to continuous norepinephrine administration to $>0.2 \mu \mathrm{g} / \mathrm{kg} / \mathrm{min}$, pericardial drainage was performed (108 min after hospital arrival). Immediately after the pericardial drainage, her blood pressure increased with infusing vasopressors. The pericardial drain was clamped after removing approximately the initial $100 \mathrm{ml}$ of pericardial effusion. Because pericardial drainage improved hemodynamics, additional closure of the 


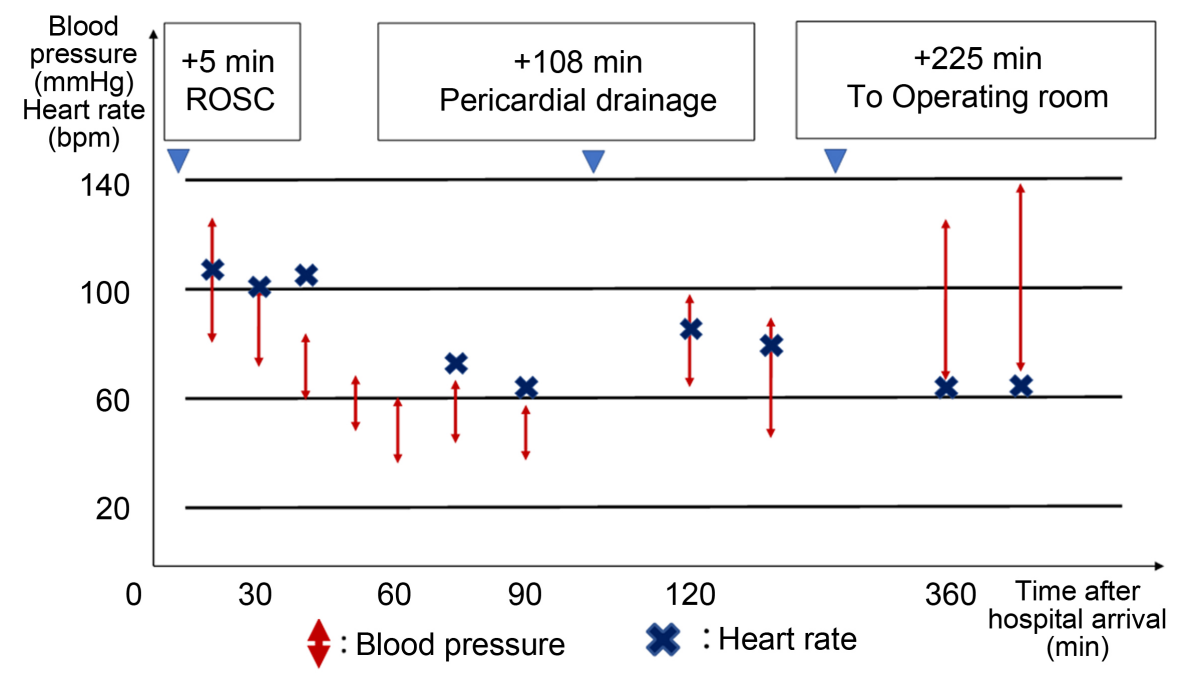

Figure 1. Hemodynamics after hospital arrival. Return of spontaneous circulation (ROSC) was achieved 5 min after hospital arrival (maximum cardiac arrest time, $27 \mathrm{~min}$ ). Hemodynamics remains unstable after ROSC; thus, pericardial drainage was performed for cardiac tamponade. After a small amount of pericardial drainage, hemodynamics was stabilized under a vasopressor support. Then, TEVAR was performed in the operating room.
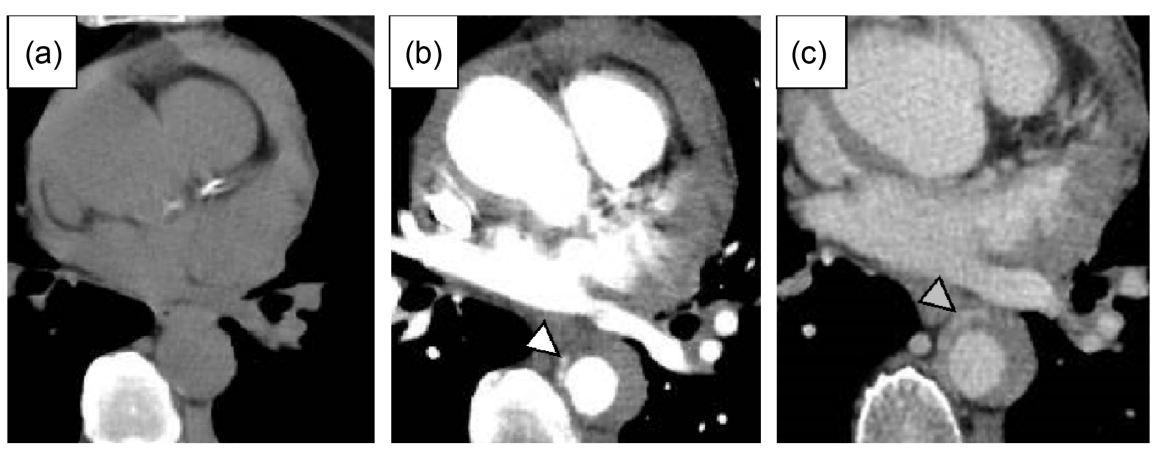

Figure 2. Findings of triple-phase computed tomography (CT) scans of the chest in the emergency room. Plain CT (a) showed no crescent signs with high density in the descending thoracic aorta. Pericardial fluid was recognized. Leakage of contrast medium (white triangle) was observed in the descending aorta in the arterial phase (b), and further metastasis (gray triangle) was observed in the equilibrium phase (c). The shape of the false lumen was concave. Based on these findings, the patient was diagnosed with retrograde Stanford type A aortic dissection with entry in the descending thoracic aorta.

entry tear using TEVAR in the descending aorta was considered to stabilize the condition. Then, TEVAR was successfully performed at $225 \mathrm{~min}$ after hospital arrival. The stent graft was deployed from the distal left subclavian artery to the descending aorta to seal the false lumen entry (Figure 3). All procedures were completed without major complications. The amount of pericardial drainage was not significantly increased $(25 \mathrm{ml} / 12 \mathrm{~h})$ and her hemodynamics improved after TEVAR. As CT scan on the 3rd hospital day showed no false lumen enlargement, the pericardial drain was removed on the 4 th hospital day. The patient was extubated on the 13th hospital day. The patient finally recovered speaking some greeting words. Follow-up CT on the 33rd hospital day showed 


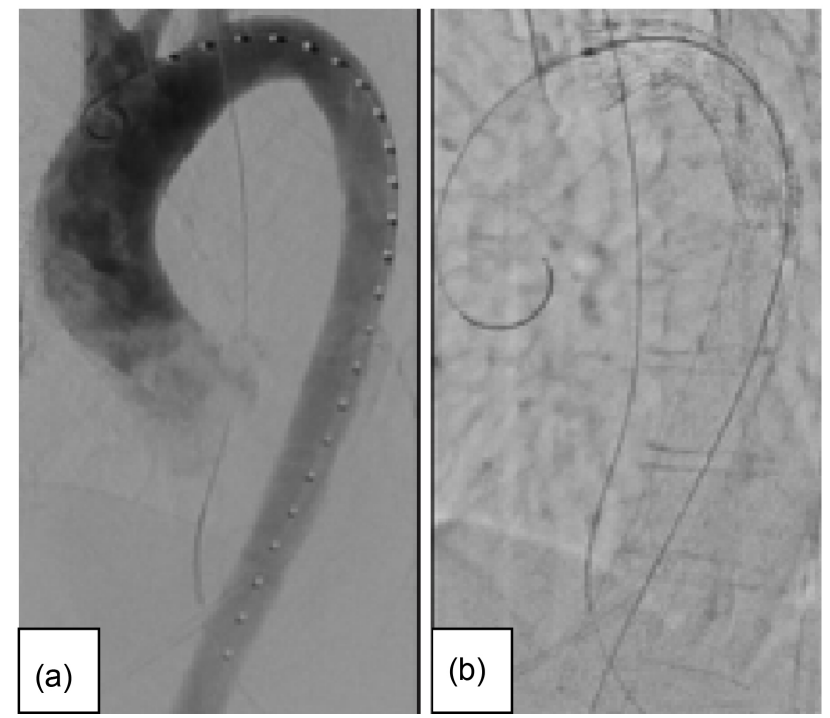

Figure 3. Intraoperative digital subtraction angiograms (DSA). The entry tear was not found in the descending aorta at the time of intraoperative DSA (a). Post-deployment angiography (b) showed that stent grafting was deployed from the distal left subclavian artery to the descending aorta to seal the false lumen entry.
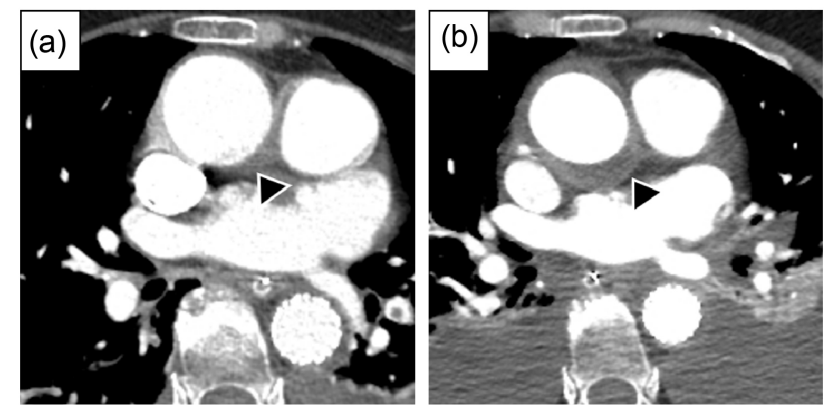

Figure 4. Follow-up CT on the 33rd hospital day (a) showed less false lumen size than that on the 3rd hospital day (b).

reduced false lumen size (Figure 4). The patient was transferred to a rehabilitation hospital on the 57th hospital day. At hospital discharge, the cerebral and overall performance categories were grade 3 .

\section{Discussion}

The patient recovered from cardiac arrest due to TAAD by TEVAR, generally assuming poor prognosis. A few cases of recovery from TAAD leading to CPA were reported [2]. The initial rhythm of the patient was considered as a key point for recovery. PEA was considered as the initial rhythm in this patient and had continued during resuscitation. PEA may have maintained weak brain circulation, whereas asystole does not have any circulation [6]. Maintained circulation may have led to patient recovery. Another reason for recovery was an adequate time from cardiopulmonary arrest to hospital arrival. Maximum cardiac 
arrest time was presumed to be only $27 \mathrm{~min}$. Furthermore, a bystander CPR started relatively soon after a cardiac arrest. Third, initial head CT showed no signs of bleeding or severe ischemia. Patients with cardiac arrest should not be entirely excluded from the intervention and be considered comprehensively including other factors.

We did not choose surgical graft replacement but TEVAR for r-TAAD due to two reasons: avoid bleeding risk by open surgery and an easy sealing of the descending aortic leakage by TEVAR for r-TAAD. Open chest surgical repair requires extracorporeal membrane oxygenation with full heparinization that has a higher risk of bleeding than TEVAR [7]. Open chest surgery cannot be selected in patients with unstable hemodynamics. The efficacy of TEVAR has been reported in patients with hemodynamically stable r-TAAD [3]. TEVAR was selected this time, and good recovery was achieved without major complications. If hemodynamics is unstable due to cardiac tamponade through the descending aortic tear, TEVAR may be an effective option, even after a cardiac arrest.

\section{Conclusion}

TEVAR can lead to a successful recovery from cardiac arrest due to r-TAAD.

\section{Acknowledgements}

An informed consent to the report was obtained from the patient's family. There is no conflict of interest.

\section{Conflicts of Interest}

The authors declare no conflicts of interest regarding the publication of this paper.

\section{References}

[1] Meron, G., Kürkciyan, I., Sterz, F., Tobler, K., Losert, H., Sedivy, R., Laggner, A.N. and Domanovits, H. (2004) Non-Traumatic Aortic Dissection or Rupture as Cause of Cardiac Arrest: Presentation and Outcome. Resuscitation, 60, 143-150. https://doi.org/10.1016/j.resuscitation.2003.10.005

[2] Sugawara, Y., Kurihara, T., Ohtsubo, S., Takamatsu, M., Sasao, K., Takebe, M., Irino, S., Takahashi, M., Hirotani, T. and Sekine, K. (2016) Recovery from Out-of-Hospital Cardiopulmonary Arrest Due to Type A Acute Aortic Dissection: A Case Report. Case Reports in Clinical Medicine, 5, 505-510. https://doi.org/10.4236/crcm.2016.511064

[3] Emily, P., Andreas, W., Eric, P., Arnar, G., Christian, O., Anders, A., Simon, F., Jarmo, G., Emma, C.H., Vibeke, H., Ari, M., Shahab, N., Anders, W., Igor, Z., Tomas, G. and Anders, J. (2019) Outcome after Type A Aortic Dissection Repair in Patients with Preoperative Cardiac Arrest. Resuscitation, 144, 1-5. https://doi.org/10.1016/j.resuscitation.2019.08.039

[4] JCS Joint Working Group (2013) Guidelines for Diagnosis and Treatment of Aortic Aneurysm and Aortic Dissection (JCS 2011): Digest Version. Circulation Journal, 77, 789-828. https://doi.org/10.1253/circj.CJ-66-0057

[5] Takatoshi, H., Noriyuki, K., Ken, N., Shuji, C., Takashi, H., Takafumi, O., Toshiya, 
T., Yasumi, M., Toru, M., Satoshi, T., Naoki, Y., Hisato, I. and Hajime, S. (2019) Thoracic Endovascular Aortic Repair for Retrograde Type A Aortic Dissection. Journal of Vascular Surgery, 69, 1685-1693.

https://doi.org/10.1016/j.jvs.2018.08.193

[6] Jennifer, R., Theodore, Q., Keith, B. and Ali, P. (2020) Pseudo-Pulseless Electrical Activity in the Emergency Department, an Evidence Based Approach. American Journal of Emergency Medicine, 38, 371-375.

https://doi.org/10.1016/j.ajem.2019.158503

[7] Liu, J., Xia, J., Yan, G., Zhang, Y., Ge, J. and Cao, L. (2019) Thoracic endovascular Aortic Repair versus Open Chest Surgical Repair for Patients with Type B Aortic Dissection: A Systematic Review and Meta-Analysis. Annals of Medicine, 51, 360-370. https://doi.org/10.1080/07853890.2019.1679874 